\title{
Noise Induced Hearing Loss with Tinnitus: Does TRT Help?
}

\author{
Nidhi Vohra Maggon, ${ }^{1}$ Ashwani Sethi, ${ }^{1}$ Atul Gupta ${ }^{1}$
}

\begin{abstract}
Introduction
To determine if hearing augmentation and tinnitus retraining therapy (TRT) helps in cases of Tinnitus with Noise induced hearing loss (NIHL) and does degree of hearing loss, severity or duration of tinnitus affect recovery Materials and Methods

A prospective study was done on 100 patients of NIHL with tinnitus from Jan 14-Jul 15. Degree of hearing loss was assessed. Tinnitus severity was scored on Tinnitus handicap inventory (THI) scale as Slight, Mild, Moderate, severe or catastrophic and patients were subjected to TRT. Patients scored after 1 year of TRT. A relation between tinnitus severity, duration and degree of hearing loss on recovery from tinnitus was analysed.

$\underline{\text { Results }}$

62 of the 100 patients improved following TRT.

Discussion

In 100 patients THI scores improved from a mean of 63.12 (SD-21.12) to 38.16 (SD-18.21). Mean difference between pre and post-intervention THI scores was 24.96 (SD-17.97). Improvement was significant in severe or profound hearing loss $(P<.001)$. Tinnitus severity was slight, mild, moderate, severe or catastrophic on THI. Following TRT, $82.35 \%$ with Catastrophic, 70.96\% with severe, $52.63 \%$ with moderate, $20 \%$ with mild tinnitus improved. 1 patient with slight tinnitus did not improve. Based on duration of tinnitus three groups made; 0-6 months, 6-12 months and $>12$ months. All groups showed improvement. Reduction in Post-TRT THI was significant but did not show any difference among groups.

Conclusion

TRT helps in tinnitus with NIHL particularly if hearing loss is severe. Severe or catastrophic tinnitus patients experience greater improvement. Duration of tinnitus has no impact.

Keywords

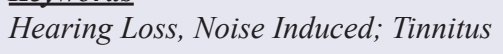

ABSTRACT

$\mathrm{T}$ innitus is considered as a phantom sound. It was documented as early as 2-3 millennium BC in dictionary of pharaonic medicine. ${ }^{1}$ Many wellknown personalities have suffered from distressing symptom including Sappho, Goya, Beethoven and Joseph Toynbee (father of Otology in England). Tinnitus is a type of Dysacusis and could be an objective tinnitus (also called somatosounds- due to an activity outside the auditory system) or subjective tinnitus. The prevalence of tinnitus in general adult population is 10.1 to $14.5 \%{ }^{2}$ but the number of people suffering due to presence of tinnitus is much less at $7.2 \% .{ }^{3}$ Various disease processes that may be associated with tinnitus are disorders of ear, neurological, bone and joints, endocrine, mental disorders, cardiovascular diseases and ototoxicity. ${ }^{4} \mathrm{Of}$ these multiple etiologies the most common pathologies associated with tinnitus are acoustic trauma, Meniere's disease and presbyacusis. ${ }^{5}$

Acoustic trauma leading to Noise induced hearing loss (NIHL) is very common in military service due to frequent exposure to very loud sounds during weapon training/use and tinnitus is a commonly associated symptom. The incidence of tinnitus in acute noise trauma is $100 \%$ and in chronic noise trauma it is $50-90 \%{ }^{6}$ NIHL with associated tinnitus or other symptoms like hyperacusis and misophonia (negative emotional response to trigger sounds) can lead to many

1 - Dept. of Otorhinolaryngology, Army College of Medical Sciences, Base Hospital, Delhi Cantonment, New Delhi

\section{Corresponding author:}

Dr Nidhi Vohra Maggon

email: magnidhi@rediffmail.com 
distressing physiological and behavioral symptoms. This results in distressing difficulty in conversation and severely affects the quality of life of these young soldiers.

We carried out a prospective study at a tertiary care military hospital where we studied 100 patients suffering from NIHL associated tinnitus and treated them with hearing augmentation and modified tinnitus retraining therapy (TRT).

\section{Materials and Methods}

A prospective study was carried out on patients who presented with NIHL with tinnitus at a tertiary care military hospital from Jan 2014 to July 2015. A total of 100 patients, all males, in the age group 23 to 42 years were enrolled in the study. Individuals who developed NIHL following exposure to loud noise and also had associated unilateral or bilateral tinnitus were included.

Exclusion criteria included patients with middle ear pathology, presbyacusis, history of hypertension and diabetes mellitus, history of exposure to ototoxic drugs and history of head-trauma.

After an informed consent for participation, all patients underwent a thorough ENT examination. Hearing assessment was done using pure tone and impedance audiometry to assess the degree and type of hearing loss. Severity of tinnitus was scored according to the Tinnitus handicap inventory (THI) scale. THI is a 25 item self-report questionnaire introduced by Newman et al (1996) that has functional, emotional and catastrophic subscales. ${ }^{7}$ It has specific questions to assess the difficulties faced by the patient due to tinnitus. A typical patient takes approximately 10 minutes to complete the THI and it is completed in presence of an expert who provides an explanation, if required. Scoring THI in a patient takes 5-10 min with a score of 4 given for a 'yes', 2 for 'sometimes' and 0 for 'no'. The THI score ranges from 0 to 100 where a higher score indicates more distress. Though the test was developed in USA but can be used in an Indian setting without any modification.
The THI scores are graded as follows:

$\begin{array}{lll}\text { - } & \text { Slight } & 0-16 \\ \text { - } & \text { Mild } & 18-36 \\ \text { - } & \text { Moderate } & 38-56 \\ \text { - } & \text { Severe } & 58-76 \\ \text { - } & \text { Catastrophic } & 78-100\end{array}$

After establishing the grade of tinnitus on THI score, patients were subjected to TRT. Since all our patients belonged to Jastreboff category $2,{ }^{8}$ they were all provided with bilateral digital hearing aids. An ENT surgeon and the speech therapist counselled them every week about the pathophysiology of tinnitus development following noise trauma and its consequences. The patients were then scored on THI after 1 year of TRT.

An analysis was done to study a relation between tinnitus severity and the degree of hearing loss. The effect of TRT on the tinnitus profile of the patient was also analysed. The results were statistically tested using statistical package for the social science system version SPSS 17.0. Continuous variables were presented as mean $\pm \mathrm{SD}$, and categorical variables are presented as absolute numbers and percentage. The comparison of normally distributed continuous variables from prepost TRT was performed using Paired t test. $\mathrm{P}<0.05$ was considered statistically significant.

\section{Results}

Total no. of patients enrolled in the study were 100 and were aged between 23 to 42 years (mean $=31.01$, $\mathrm{SD}=4.24$ ). They all underwent a standard audiological assessment and were subjected to TRT.

On basis of degree of hearing loss, they were categorized into 4 categories. 10 (10\%) individuals had slight hearing loss and 16, 45, 29 patients had moderate, severe or profound hearing loss respectively (Table I). 62 of the 100 patients showed improvement in their subjective symptoms (62\%) (Table II) and THI scores improved from a mean score of 63.12 (SD-21.12) to 38.16 (SD-18.21). The mean difference between preintervention and post-intervention THI scores was 24.96 (SD-17.97) and was statistically significant $(\mathrm{p}<0.001)$ (Table III). However, the improvement was significant 
Table I : Frequency distribution based on degree of hearing loss

\begin{tabular}{|c|c|c|}
\hline $\begin{array}{c}\text { DEGREE OF } \\
\text { HEARING } \\
\text { LOSS }\end{array}$ & FREQUENCY & PERCENTAGE \\
\hline Slight & 10 & $10.0 \%$ \\
\hline Moderate & 16 & $16.0 \%$ \\
\hline Severe & 45 & $45.0 \%$ \\
\hline Profound & 29 & $29.0 \%$ \\
\hline Total & 100 & $100 \%$ \\
\hline
\end{tabular}

only in those who suffered severe or profound hearing loss and was not significant in those who had only a slight or moderate pre-intervention hearing loss (Table IV).

Patients were categorized as suffering from slight, mild, moderate, severe or catastrophic degree of tinnitus based on THI and there were 1, 15, 19, 31, 34 patients in each category respectively (Table V). Following intervention, 28 of the 34 patients with Catastrophic tinnitus improved $(82.35 \%), 22$ of the 31 patients suffering from severe tinnitus improved (70.96\%), 10 of the 19 with moderate tinnitus improved (52.63\%) while only 3 of the 15 patients with mild tinnitus (20\%) improved and the only patient with slight tinnitus did not report improvement (Table VI).

Finally, patients were arbitrarily classified on basis of duration of tinnitus to explore the possibility of

Table II : Percentage of patients who improved after TRT

\begin{tabular}{|c|c|c|}
\hline $\begin{array}{c}\text { THI } \\
\text { CHANGE }\end{array}$ & FREQUENCY & PERCENTAGE \\
\hline Change & 62 & $62.0 \%$ \\
\hline No change & 38 & $38.0 \%$ \\
\hline Total & 100 & $100 \%$ \\
\hline
\end{tabular}

Table III : Pre-intervention and post-intervention THI scores

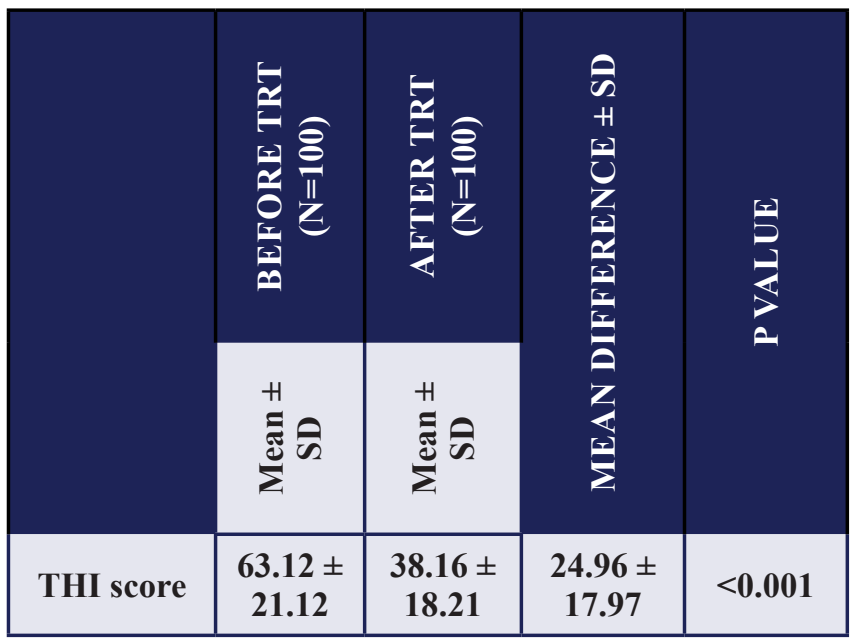

its impact on recovery from tinnitus following TRT. Temporal classification had three groups based on duration of tinnitus 0-6 months, 6-12 months and more than 12 months. 21, 50 and 29 patients were found to be there in each temporal group respectively. In the first group (0-6 months) the mean improvement in terms of THI following TRT was 25.43 (SD-21.69), in the second group (6-12 months) 22.72 (SD-16.36) and in the third group (12-24 months) 28.48 (SD-17.74). All these differences between pre-TRT and post-TRT THI values were statistically significant but did not show any significant difference amongst the group.

\section{Discussion}

Tinnitus is defined as an auditory perception due to aberrant spontaneous activity arising from an altered state of excitation or inhibition within auditory system. The auditory system operates by a complex feedback phenomenon which includes an ascending afferent pathway and a descending efferent pathway. It's also connected with central nervous system through other non-auditory structures like limbic system, reticular activating system serotonergic, somato-sensory and hypothalamic system. ${ }^{9}$

The pathophysiology of tinnitus in NIHL is complex and is thought to result from various pathological processes. Some of them are explained here. 
Table IV: Pre-intervention and post-intervention THI scores based on degree of hearing loss

\begin{tabular}{|c|c|c|c|c|c|}
\hline $\begin{array}{c}\text { DEGREE OF } \\
\text { HEARING } \\
\text { LOSS }\end{array}$ & $\mathrm{N}$ & BEFORE TRT & AFTER TRT & \multicolumn{2}{c|}{ MEAN } \\
\cline { 2 - 5 } Slight & 10 & $36.40 \pm 10.26$ & $22.20 \pm 15.45$ & $14.20 \pm 14.89$ & P VALUE \\
\hline Moderate & 16 & $46.75 \pm 20.95$ & $33.88 \pm 20.93$ & $12.88 \pm 13.08$ & 0.015 \\
\hline Severe & 45 & $68.62 \pm 16.79$ & $40.44 \pm 15.51$ & $28.18 \pm 18.46$ & $<.001$ \\
\hline Profound & 29 & $72.83 \pm 14.56$ & $42.48 \pm 18.82$ & $30.34 \pm 16.48$ & $<0.001$ \\
\hline
\end{tabular}

Following acoustic trauma, pathological changes in cochlea cause reduced auditory input leading to compensatory disinhibition in proximal auditory pathway. This disinhibition results in tonotopic reorganization that, in turn, leads to tinnitus. Another pathological process implicated in NIHL, is down regulation of Gamma amino butyric acid (GABA) in auditory periphery which leads to reduced inhibitory function of the efferent pathway. ${ }^{10}$ An alteration in the spontaneous activity and plastic transformation of the brain results from the reduced afferent input and compensatory disinhibition in proximal auditory pathways ${ }^{11}$ leading to tinnitus.

Cochlear lesions lead to reduced spontaneous activity in auditory nerve and ventral cochlear nucleus ${ }^{12}$ and an increased activity in dorsal cochlear nucleus, inferior colliculus, medial geniculate body and cortical

Table V : Frequency distribution of patients based on degree of tinnitus

\begin{tabular}{|c|c|c|}
\hline $\begin{array}{c}\text { DEGREE OF } \\
\text { TINNITUS }\end{array}$ & $\begin{array}{c}\text { NO. OF } \\
\text { PATIENTS }\end{array}$ & PERCENTAGE \\
\hline Slight (Sligh) & 1 & $1 \%$ \\
\hline Mild & 15 & $15 \%$ \\
\hline Moderate(Mod) & 19 & $19 \%$ \\
\hline Severe & 31 & $31 \%$ \\
\hline Catastrophic (Cat) & 34 & $34 \%$ \\
\hline
\end{tabular}

neurons. ${ }^{13}$ This neuronal hyperactivity is thought to be the neurophysiological correlate of clinical tinnitus thus demonstrating that tinnitus occurs due to central processing. ${ }^{14}$

Various treatment modalities have been tried to treat tinnitus because a fully satisfactory treatment still remains elusive. ${ }^{15}$ These treatment modalities include :

- Pharmacotherapy

- Cognitive behavior therapy

- $\quad$ Tinnitus masking

- $\quad$ TRT

- Trans-cranial electric or magnetic stimulation, etc.

Pharmacotherapy includes many different classes of drugs like antidepressants, GABA analogues, Cachannel antagonists, anti-epileptics, prostaglandin analogues, lidocaine etc.

Lidocaine is one of the most promising drugs for control of tinnitus and probably acts by sodium channel blockage. ${ }^{16}$. Its use is impractical due to intra-venous mode of delivery. In some studies, it has been used successfully by a Trans-tympanic application. In a study by Sakata $\mathrm{H}$ et al a Trans tympanic infusion of $4 \%$ lidocaine was given in cases of cochlear tinnitus associated with vestibular symptoms and a success rate of $81 \%$ was achieved with this therapy. ${ }^{17}$

Gingko biloba, an extract from the leaves of maidenhair plant has been used commonly for tinnitus but its efficacy has not been proved in randomized controlled trials. ${ }^{18}$

Psychotherapy for tinnitus includes cognitive 
Table VI : Category-wise improvement on basis of degree of tinnitus

\begin{tabular}{|c|c|c|}
\hline $\begin{array}{c}\text { THI } \\
\text { CHANGE }\end{array}$ & FREQUENCY & PERCENTAGE \\
\hline Cat-Cat & 6 & $6.0 \%$ \\
\hline Cat-Mild & 12 & $12.0 \%$ \\
\hline Cat-Mod & 11 & $11.0 \%$ \\
\hline Cat-Sev & 4 & $4.0 \%$ \\
\hline Cat-Slight & 1 & $1.0 \%$ \\
\hline Sev-Sev & 9 & $1.0 \%$ \\
\hline Sev-Mild & 10 & $10.0 \%$ \\
\hline Sev-Mod & 10 & $10.0 \%$ \\
\hline Sev-Slight & 2 & $9.0 \%$ \\
\hline Mod-Mild & 8 & $8.0 \%$ \\
\hline Mod-Mod & 9 & $9.0 \%$ \\
\hline Mod-Slight & 2 & $2.0 \%$ \\
\hline Mild-Slight & 3 & $3.0 \%$ \\
\hline Mild-Mild & 12 & $12.0 \%$ \\
\hline Slight-Slight & 1 & $1.0 \%$ \\
\hline Total & 100 & $100 \%$ \\
\hline
\end{tabular}

behavior therapy (CBT) and biofeedback. Hallam et al. ${ }^{16-19}$ introduced CBT which is based on the psychological model of tinnitus. In this, the negative emotions generated by tinnitus are separated from its perception. Its efficacy has been proved by eight small randomized control trial ${ }^{20}$ and also by a review of literature by Cima et al. ${ }^{21}$

Tinnitus maskers have been used for reduction or abolition of tinnitus by sound presentation devices (pillow speaker, MP 3 players) placed either 'behind' or 'in' the ear. They yield a positive result on the negative effects of tinnitus ${ }^{22}$ but accurate data regarding their efficacy is not available though they are claimed to provide immediate relief in contrast to TRT. ${ }^{23}$

Trans cranial magnetic stimulation at different frequencies has been also been tried for tinnitus and an objective evaluation done by using THI or self-rating. It has been concluded that daily repeated sessions may be useful for tinnitus. ${ }^{24}$

TRT is based on neurophysiological model of tinnitus. ${ }^{25}$ This model suggests that the auditory system has only a secondary role in the distress associated with tinnitus. The distress caused by tinnitus occurs when the auditory activity spreads to the limbic and autonomic system, especially the sympathetic autonomic system. The aim of TRT is to educate the patient regarding tinnitus, try to achieve habituation and remove the negative emotions associated with tinnitus perception.

Jastreboff has divided tinnitus patients in five categories (0-4) on basis of

a. impact of tinnitus on patient's life

b. associated hearing loss perceived by patients

c. problem in sound tolerance

d. effect of exposure to moderate to loud sounds on symptoms

All our patients belonged to Jastreboff Category 2 (tinnitus associated with hearing loss) and were managed with counselling and digital hearing aids.

Clinically distressing tinnitus appears more frequently when the initiating event is associated with high noise level in a setting of background emotional stress as occurs in professions like military, firefighters and policemen etc. In such populations the tinnitus is associated with NIHL and various studies have shown a success rate of $60-70 \%$ with use of hearing aid and TRT. $^{26}$

In our study the patient profile was young male patients (age ranging from 23-42years) who were exposed to loud sounds of gun and artillery fire (sound ranging $156-180 \mathrm{~dB}$ SPL) They presented to us after varying periods of time after the noise exposure. By use of hearing aids and TRT in form of repeated counselling we achieved a success of $62 \%$ in reducing distress associated with tinnitus and also improved hearing 
acuity. A study conducted by Bartnik $G$ et al evaluated effect of TRT in patients with tinnitus and subjective hearing loss versus those who had tinnitus only. They evaluated 40 patients and found that $90 \%$ of patients with tinnitus and subjective hearing loss had significant improvement at after 1 year of therapy. ${ }^{27}$ This study has not categorized the patients on basis of degree of hearing loss whereas in our study we have quantified the degree of hearing loss and have assessed the effect of TRT on various categories independently.

A Cochrane review to determine the efficacy of TRT for management of tinnitus was carried out by John S Philips which concluded that THI improved in the range of 18.2-50.4 points this wide variation being the result of considering patients with varying degree of severity of tinnitus as different categories. ${ }^{28}$ We found an average improvement in THI score of $24.96 \pm 17.97$ (Table III). However, this study also did not take in to account the accompanying hearing loss. Baracca GN in a study of 51 patients found that patients who had suffered from tinnitus for less than one year achieved significantly better results than patients who had suffered for a longer period of time and concluded that TRT is an effective tool in the treatment of tinnitus. ${ }^{29}$ In our study, the duration of tinnitus did not influence the extent of improvement in THI scores following TRT probably because the maximum duration of tinnitus in our cohort was only 2 years, also the patients in other studies did not suffer from NIHL which was addressed by amplification in present study.

In another study by Henry et al in 2006 revealed no effect of the duration of tinnitus on the effectiveness of TRT. ${ }^{30}$ It also stated that degree of hearing loss too, had no impact on TRT results unlike our results where the effect of TRT in cases of slight and moderate hearing loss was notstatistically significant.at $p<.001$ (However, it would have been significant if we had taken $\mathrm{p}<.05$ and therefore the results are inherently not very different).

There was no improvement in $38 \%$ of our patients and this was probably because THI scores are influenced by underlying emotional stress. Most of our patients being young soldiers and having been evacuated from active military zones had high level of anxiety. This may explain a comparatively modest success rate in our study.

\section{Conclusion}

Tinnitus frequently accompanies NIHL and adds to patient morbidity. TRT has been found to be effective in reducing this morbidity. However, its multifactorial causation and varied degree of severity makes it difficult to predict possible improvement as also the influence of various factors on degree of improvement. We conducted a study on 100 patients and noticed improvement in 62 of them on basis of a measurable and objective questionnaire based parameter, the THI score. Improvement was statistically significant in cases of severe or profound hearing loss $(\mathrm{P}<.001)$.

Tinnitus was classified on basis of its severity into slight, mild, moderate, severe or catastrophic on THI score. Following TRT, $82.35 \%$ patients with Catastrophic, $70.96 \%$ with severe, $52.63 \%$ with moderate, $20 \%$ with mild tinnitus showed significant improvement. 1 patient with slight tinnitus did not improve. More severe tinnitus showed greater amelioration of symptoms after TRT.

Based on the duration of tinnitus three groups made; 0-6 months, 6-12 months and $>12$ months. All groups showed improvement. However, the reduction in PostTRT THI scores was significant in all three groups but did not show any difference amongst the 3 groups thus indicating that duration of tinnitus did not affect the prognosis.

\section{References}

1. Stephens SDG. The treatment of tinnitus-Ahistorical perspective. Journal of Laryngology and Otology.1984;98:963-72

2. Davies A, Rafaie EA. Epidemiology of tinnitus. In: Tyler R S (ed.) Tinnitus Handbook. Singular, San Diego, CA, 2000:1-23

3. Smith P, Coles R. Epidemiology of tinnitus: An update. In: FeldmannH(ed.). Proceedings of the third international tinnitus seminar, Munster. Karlsruhe: Harsch Verlag,1987:147-53

4. Axelsson A. Causes of tinnitus. In: Aran JM, Dauman R (eds). Tinnitus 91. Proceedings of IV international tinnitus seminar, Bordeaux. Amsterdam/New York: Kugler publications, 1992:275-7

5. Nicolas Puel C, Faulconbrodge RL, Guitton M, Puel JL, Mondain M, Uziel A. Characteristics of tinnitus and etiology of associated hearing loss: a study of 123 patients. International Tinnitus Journal. 2002; 8:37-44

6. Dias Adriano, Cordeiro Ricardo, Corrente José Eduardo, Gonçalves Cláudia Giglio de Oliveira. Association between 
noise-induced hearing loss and tinnitus. Cad. Saúde Pública [Internet]. 2006 Jan [cited 2017 Jan 16] ; 22( 1 ): 63-68

7. Newman CW1, Jacobson GP, Spitzer JB. Development of the Tinnitus Handicap Inventory. Arch Otolaryngol Head Neck Surg. 1996 Feb;122(2):143-8

8. Henry JA, Jastreboff MM, Jastreboff PJ, Schechter MA, Fausti SA. Guide to conducting tinnitus retraining therapy initial and follow-up interviews. J Rehabil Res Dev. 2003 MarApr;40(2):157-77

9. LeDoux JE, Sakaguchi A, Reis DJ. Sub cortical efferent projections of the medial geniculate nucleus mediate emotional response conditioned to acoustic stimuli. Journal of Neuroscience. 1983;4:683-98

10. Caspary DM, Salvi RJ, Helfert RH, Brozoski TJ , Bauer CA. Neuropharmacology of noise induced hearing loss in brainstorm auditory structures. In: HendersonD, Prasher D et al(eds). Noise induced hearing loss: Basic mechanisms, prevention and control. Noise Research Network Publications, London, 2001:169-83

11. Eggermont JJ .Physiological mechanisms and neural models. In: TylerR (Ed.). Tinnitus Handbook. San Diego : Singular, 2000:85-122

12. Wang J, Ding D, Salvi RJ. Functional reorganization in chinchilla inferior colliculus associated with chronic and acute cochlear changes. Hearing research. 2002;168:238-49

13. Komiya H, Eggermont JJ. Spontaneous firing activity of cortical neurons in adult cats with reorganized tonotopic map following pure tone trauma. Otolaryngologica. 2000;120:750-6

14. Zacharek MA, Kaltenbach JA, Mathog TA, Zhang J. Effects of cochlear ablation on noise induced hyperactivity in the hamster dorsal cochlear nucleus: implication for the origin of noise induced tinnitus. Hearing Research.2002; 172:137-44

15. The Cochrane Library/Ear Nose Throat disorders/Ear/Inner ear/Tinnitus. John S Phillips, Don McFerran. First published: 17 March 2010. Editorial Group: Cochrane ENT Group DOI: 10.1002/14651858.CD007330.pub2

16. Martin FW, Colman BH. Tinnitus : A double blind cross over controlled trial to evaluate the use of lignocaine. Clinical Otolaryngology. 1980; 5:3-11

17. Laurikainen EA, Johansson RK, Kileny PR. Effects of intratympanically delivered lidocaine on the auditory system in humans. Ear and Hearing. 1996; 17:49-54

18. 185. Dobie RA. A review of randomized clinical trial in tinnitus. Laryngoscope.1999; 109:1202-11

19. Hallam RS, RachmanS, Hinchcliffe R. Psychological aspects of tinnitus. In: Rachman S (ed). Contribution to medical psychology, Vol 3. Oxford: Pergamon Press, 1984:31-4

20. Anderson G, Lyttkens L.A meta- analytic review of Psychological treatments for tinnitus. British Journal of Audiology. 1999;33:201-210

21. Cima RF, Andersson G, Schmidt CJ, Henry JA. Cognitivebehavioral treatments for tinnitus: a review of the literature. J Am Acad Audiol. 2014 Jan;25(1):29-61. doi: 10.3766/ jaaa.25.1.4

22. Vernon JA, Meikle MB.Tinnitus masking. In: Tyler R(ed) Tinnitus handbook .San diego :Singular, 2000:313-76

23. Vernon JA. Pathophysiology of tinnitus: A special casehyperacusis and a proposed treatment. Am J Otol 1987;8: 201-2

24. E M Khedr, J C Rothwell, M A Ahmed, A El-Atar. J Neurol Neurosurg Psychiatry 2008;79:212-215

25. Jasterboff PJ, Hazell JWP. A neurophysiological approach to tinnitus: Clinical implications. British journal of Audiology. 1993;27:7-17

26. McKinney CJ.An evaluation of the TRT method. In: Hazell JWP(ed) Proceedings of the sixth international tinnitus seminar, Cambridge, UK. London: The tinnitus and hyperacusis Centre, 199:99-105

27. Bartnik G, Fabijańska A, Rogowski M. Effects of tinnitus retraining therapy (TRT) for patients with tinnitus and subjective hearing loss versus tinnitus only. Scand Audiol Suppl. 2001 ; ( 52):206-8

28. Phillips JS1, McFerran D. Tinnitus Retraining Therapy (TRT) for tinnitus. Cochrane Database Syst Rev. 2010 Mar 17;(3):CD007330. doi: 10.1002/14651858.CD007330.pub2

29. Baracca GN et al (2007) Results of tinnitus retraining therapy after 18 months: Our experience. Int J Audiol 46(5):217-222

30. Henry JA, Schechter MA, Zaugg TL, Griest S, Jastreboff PJ, Vernon JA, Kaelin C, Meikle MB, Lyons KS, Stewart BJ. Clinical trial to compare tinnitus masking and tinnitus retraining therapy. Acta Otolaryngol Suppl. 2006 Dec;(556):64-9. 